\title{
ON THE OSOFSKY-SMITH THEOREM*
}

\author{
SEPTIMIU CRIVEI \\ Faculty of Mathematics and Computer Science, "Babeş-Bolyai" University, \\ Str. M. Kogălniceanu 1, 400084 Cluj-Napoca, Romania \\ e-mail: crivei@math.ubbcluj.ro \\ CONSTANTIN NĂSTĂSESCU \\ Faculty of Mathematics and Computer Science, University of Bucharest, \\ Str. Academiei 14, 010014 Bucharest, Romania \\ e-mail: cnastase@al.math.unibuc.ro \\ and BLAS TORRECILLAS \\ Departamento de Álgebra y Análisis, Universidad de Almería, 04071 Almería, Spain \\ e-mail: btorreci@ual.es
}

\begin{abstract}
We recall a version of the Osofsky-Smith theorem in the context of a Grothendieck category and derive several consequences of this result. For example, it is deduced that every locally finitely generated Grothendieck category with a family of completely injective finitely generated generators is semi-simple. We also discuss the torsion-theoretic version of the classical Osofsky theorem which characterizes semisimple rings as those rings whose every cyclic module is injective.
\end{abstract}

2002 Mathematics Subject Classification. 16D50, 16S90.

1. Introduction. In the late 1960s, Osofsky showed her classical result which asserts that a ring is semi-simple if and only if every cyclic module is injective [8, Theorem], [9, Corollary]. Among the categorical generalizations of the Osofsky theorem, we mention the version established by Gómez Pardo et al. [5]. They showed that if $\mathcal{C}$ is a locally finitely generated Grothendieck category and $M$ is a finitely presented object of $\mathcal{C}$ which is completely (pure-)injective and has a von Neumann regular endomorphism ring $S$, then $S$ is a semi-simple ring [5, Theorem 1]. In the early 1990s, Osofsky and Smith established a module counterpart of the original Osofsky theorem. They proved that if $M$ is a cyclic module with the property that every cyclic submodule of $M$ is completely extending, then $M$ is a finite direct sum of uniform modules [10]. As a consequence, if $M$ is a module with every quotient of a cyclic submodule injective, then $M$ is semi-simple. In the same paper, Osofsky and Smith noted that their result still holds in a more general categorical setting.

The purpose of this paper is to discuss some categorical version of the OsofskySmith theorem and give several applications. We first consider the setting of a locally finitely generated Grothendieck category $\mathcal{C}$ and deduce that if $\mathcal{C}$ has a family of completely injective finitely generated generators, then $\mathcal{C}$ is semi-simple. As an application, we give a positive partial answer to the following question raised by

${ }^{*}$ To Professor Patrick F. Smith on the occasion of his 65 th birthday. 
M. Teply: Does the torsion-theoretic version of the Osofsky theorem hold? In other words, if $\tau$ is a hereditary torsion theory such that every cyclic module is $\tau$-injective, does it follow that every module is $\tau$-injective? Finally, we show that a ring is semisimple if and only if every cyclic module is $\tau$-injective $\tau$-complemented.

\section{Locally finitely generated Grothendieck categories.}

Definition 2.1. Let $\mathcal{C}$ be a Grothendieck category. Then an object $C$ of $\mathcal{C}$ is called completely injective if for every object $M$ of $\mathcal{C}$ and every morphism $f: C \rightarrow M, \operatorname{Im}(f)$ is an injective object.

REMARK. As an immediate consequence of the existence of an injective hull for every object in $\mathcal{C}$, an object $C$ of $\mathcal{C}$ is completely injective if and only if for every injective object $M$ of $\mathcal{C}$ and every morphism $f: C \rightarrow M, \operatorname{Im}(f)$ is an injective object.

We begin with a property that will be needed later.

PROPOSITION 2.2. Let $\mathcal{C}$ be a Grothendieck category and $\left(U_{i}\right)_{i \in I}$ a family of completely injective objects of $\mathcal{C}$. Then every finite direct sum of $U_{i}$ 's is completely injective.

Proof. Consider a finite direct sum of $U_{i}$ 's, say $U_{1} \oplus \cdots \oplus U_{n}$, and let $f: U_{1} \oplus$ $\cdots \oplus U_{n} \rightarrow M$ be a morphism in $\mathcal{C}$. We show that $\operatorname{Im}(f)$ is an injective object. We prove it for $n=2$, the general case that follows by induction. Let $f: U_{1} \oplus U_{2} \rightarrow$ $M$ be a morphism in $\mathcal{C}$. Denote by $i_{1}: U_{1} \rightarrow U_{1} \oplus U_{2}$ and $i_{2}: U_{2} \rightarrow U_{1} \oplus U_{2}$ the inclusion morphisms. Also, put $f_{1}=f \circ i_{1}$ and $f_{2}=f \circ i_{2}$. Then it is easy to see that $\operatorname{Im}(f)=\operatorname{Im}\left(f_{1}\right)+\operatorname{Im}\left(f_{2}\right)$. Let $X=\operatorname{Im}\left(f_{1}\right), Y=\operatorname{Im}\left(f_{2}\right)$, and let $g: U_{1} \rightarrow X /(X \cap Y)$ be the composition of the natural epimorphisms $U_{1} \rightarrow X$ and $X \rightarrow X /(X \cap Y)$. Then $(X+Y) / Y \cong X /(X \cap Y) \cong \operatorname{Im}(g)$ is an injective object by hypothesis. But $Y$ is also injective, and so $\operatorname{Im}(f)=X+Y$ is an injective object.

Recall that a Grothendieck category $\mathcal{C}$ is called locally finitely generated if it has a family of finitely generated generators [12].

COROLLARY 2.3. Let $\mathcal{C}$ be a locally finitely generated Grothendieck category with a family of completely injective finitely generated generators. Then every finitely generated object in $\mathcal{C}$ is injective.

EXAMPLE 2.4. The conclusion of Proposition 2.2 does not hold for an infinite family. Indeed, let us consider an infinite family of fields $\left(K_{i}\right)_{i \in I}$ and let $R=\prod_{i \in I} K_{i}$. Then $R$ is a commutative von Neumann regular ring, that is, a $V$-ring, and so every simple $R$-module is injective. Now let $\left(e_{i}\right)_{i \in I}$ be the family of primitive orthogonal idempotents in $R$. Clearly, each $S_{i}=R e_{i}$ is a simple $R$-module, and so injective. Then each $S_{i}$ is actually completely injective. Also, we have $\bigoplus_{i \in I} S_{i}=\operatorname{Soc}(R)$. Clearly, $\bigoplus_{i \in I} S_{i}$ is not injective, because otherwise this would imply that $R=\operatorname{Soc}(R)$. Now if we take $M=\bigoplus_{i \in I} S_{i}$ and $f$ to be the identity homomorphism, it follows that $C=M$ is not completely injective.

EXAMPLE 2.5. If $R$ is a right hereditary ring, then it is clear that the class of completely injective objects in the category Mod- $R$ of right $R$-modules coincides with the class of injective objects in Mod- $R$.

In order to be able to state the Osofsky-Smith theorem, we need the definition of an extending object in a Grothendieck category, which is the same as for modules. 
Definition 2.6. Let $\mathcal{C}$ be a Grothendieck category. An object $M$ of $\mathcal{C}$ is called extending if every subobject of $M$ is essential in a direct summand of $M$. Equivalently, $M$ is extending if and only if every essentially closed subobject of $M$ is a direct summand of $M$.

An object $M$ of $\mathcal{C}$ is called completely extending if for every object $M$ of $\mathcal{C}$ and every morphism $f: C \rightarrow M, \operatorname{Im}(f)$ is an extending object.

Let $\mathcal{C}$ be a Grothendieck category. For a class $\mathcal{P}$ of objects of $\mathcal{C}$, by a $\mathcal{P}$-subobject we mean a subobject belonging to $\mathcal{P}$. Let $\mathcal{P}$ be a class of finitely generated objects in $\mathcal{C}$ with the following properties:

$\left(P_{1}\right) \mathcal{P}$ is closed under quotients.

$\left(P_{2}\right)$ If $X \in \mathcal{P}$ and $Y$ is a $\mathcal{P}$-subobject of a quotient object of $X$, then there is a $\mathcal{P}$-subobject $Z$ of $X$ that projects onto $Y$.

Some examples of such classes $\mathcal{P}$ in $\mathcal{C}$ are the following: the class of all finitely generated objects, the class of finitely generated semi-simple objects and any class of finitely generated objects closed under subobjects and quotients.

Now basically the same proof of the basic theorem for modules (see [7] or [10]) works in our categorical context. This has also been noted in the original paper of Osofsky and Smith [10].

THEOREM 2.7. Let $\mathcal{C}$ be a Grothendieck category. Let $\mathcal{P}$ be a class of finitely generated objects in $\mathcal{C}$ satisfying $\left(P_{1}\right)$ and $\left(P_{2}\right)$ and let $M \in \mathcal{P}$ be such that every $\mathcal{P}$-subobject of $M$ is completely extending. Then $M$ is a finite direct sum of uniform objects.

The next two corollaries are obtained as [10, Corollaries 1 and 2].

COROLLARY 2.8. Let $\mathcal{C}$ be a Grothendieck category such that every finitely generated object is extending. Then every finitely generated object is a finite direct sum of uniform objects.

Corollary 2.9. Let $\mathcal{C}$ be a Grothendieck category. Let $M$ be an object of $\mathcal{C}$ such that every quotient of every finitely generated subobject of $M$ is injective. Then $M$ is semi-simple.

Recall that a Grothendieck category $\mathcal{C}$ is called semi-simple if every object of $\mathcal{C}$ is semi-simple [12]. Now Corollaries 2.3 and 2.9 yield the Osofsky-Smith theorem in locally finitely generated Grothendieck categories, stated as follows.

THEOREM 2.10. Let $\mathcal{C}$ be a locally finitely generated Grothendieck category with a family of completely injective finitely generated generators. Then $\mathcal{C}$ is semi-simple.

By Corollary 2.3, the property of complete injectivity of the finitely generated generators of a locally finitely generated Grothendieck category passes to each finitely generated object. Now we immediately have the following consequences of Theorem 2.10.

COROLlary 2.11 [8, Theorem]. Let $R$ be a ring with identity such that every cyclic (finitely generated) module is injective. Then $R$ is semi-simple.

Corollary 2.12 [3, Corollary 7.14]. Let $R$ be a ring with identity, $M$ a module and $\sigma[M]$ the category of $M$-subgenerated modules. Suppose that every cyclic (finitely generated) module in $\sigma[M]$ is $M$-injective. Then $M$ is semi-simple. 
COROLlaRY 2.13. Let $R$ be a ring with enough idempotents such that every cyclic (finitely generated) module is injective. Then $R$ is semi-simple.

Recall that a Grothendieck category $\mathcal{C}$ is called spectral if every object of $\mathcal{C}$ is injective. It is well known that $\mathcal{C}$ is semi-simple if and only if it is locally finitely generated and spectral [12]. This suggests us to raise the following natural question, whose positive answer would generalize the Osofsky-Smith theorem 2.10.

QUESTION 1. If $\mathcal{C}$ is a Grothendieck category with a family of completely injective generators, does it follow that $\mathcal{C}$ is spectral?

3. Applications to torsion theories. Throughout this section, $R$ is a ring with identity, all modules are unitary right $R$-modules and $M$ is a module. Also, Mod- $R$ denotes the category of unitary right $R$-modules, $\sigma[M]$ denotes the full subcategory of Mod- $R$ consisting of $M$-subgenerated modules and $\tau=(\mathcal{T}, \mathcal{F})$ is a hereditary torsion theory in Mod- $R$. Recall that a submodule $B$ of a module $A$ is called $\tau$ dense (respectively $\tau$-closed) in $A$ if $A / B$ is $\tau$-torsion (respectively $\tau$-torsion free). Also, a module $M$ is called $\tau$-injective if for every module $B$ and every $\tau$-dense submodule $A$ of $B$, every homomorphism $A \rightarrow M$ extends to a homomorphism $B \rightarrow M$. For further background on torsion theories the reader is referred to [4] or [12].

Now we have the following consequence of the categorical Osofsky-Smith theorem for torsion theories.

COROLlary 3.1. Suppose that every cyclic $\tau$-torsion module is $\tau$-injective. Then every $\tau$-torsion module is $\tau$-injective.

Proof. Note that $\mathcal{T}$ is generated by the modules of the form $R / I$ for the $\tau$-dense right ideals $I$ of $R$. Each factor of such an $R / I$ is cyclic $\tau$-torsion, and hence, $\tau$ torsion $\tau$-injective by hypothesis, and so injective in $\mathcal{T}$. Thus, each such generator $R / I$ is completely injective in $\mathcal{T}$. Now by Theorem $2.10, \mathcal{T}$ is semi-simple, and so spectral. Then every $\tau$-torsion module is injective in $\mathcal{T}$, that is, every $\tau$-torsion module is $\tau$-injective.

A related question is the following one, which was raised by M. Teply:

QUESTION 2. If every cyclic module is $\tau$-injective, does it follow that every module is $\tau$-injective?

REMARK. Note that, by Corollary 3.1, if every cyclic $\tau$-torsion module is $\tau$-injective, then every $\tau$-torsion module is $\tau$-injective, and so every $\tau$-torsion module is semi-simple by [4, Proposition 8.15]. Hence, Question 2 reduces to the case of a specialization of the Dickson torsion theory [2]. Recall that the Dickson torsion theory is the hereditary torsion theory generated by all simple modules. Its torsion class consists of all semiartinian modules, whereas its torsion-free class consists of all modules with zero socle.

In the following we shall obtain a positive answer in case $\tau$ is of finite type. Recall that a torsion theory is called of finite type if its Gabriel filter contains a cofinal subset of finitely generated left ideals. A module is called $\tau$-finitely generated if it has a finitely generated $\tau$-dense submodule. We need the following lemma.

Lemma 3.2. Suppose that every cyclic module is $\tau$-injective. Then every $\tau$-finitely generated module is $\tau$-injective. 
Proof. First we show that every finitely generated module is $\tau$-injective. Let $M$ be a finitely generated module, say $M=R x_{1}+\cdots+R x_{n}$. Use induction on $n$. For $n=1$ it is clear. Suppose that every module generated by $n-1$ elements is $\tau$-injective. Then $M /\left(R x_{1}+\cdots+R x_{n-1}\right) \cong R x_{n} /\left(\left(R x_{1}+\ldots+R x_{n-1}\right) \cap R x_{n}\right)$ is cyclic, and so $\tau$ injective. But $R x_{1}+\cdots+R x_{n-1}$ is also $\tau$-injective, so that $M$ is $\tau$-injective.

Now let $M$ be a $\tau$-finitely generated module; hence, $M$ has some $\tau$-dense finitely generated submodule $N$. Then $N$ is $\tau$-injective by the argument given in the previous paragraph. Clearly, $M / N$ is $\tau$-torsion, and hence, $\tau$-injective by Corollary 3.1 . Thus, it follows that $M$ is $\tau$-injective.

THEOREM 3.3. Let $\tau$ be of finite type and suppose that every cyclic module is $\tau$ injective. Then every module is $\tau$-injective.

Proof. Let $I$ be a $\tau$-dense left ideal of $R$. Then there exists a finitely generated left ideal $J \subseteq I$ and we have $I / J \tau$-torsion. Then $J$ is $\tau$-injective by Lemma 3.2; hence, it is a direct summand of $R$, and so a direct summand of $I$, say $I=J \oplus J^{\prime}$. But $J^{\prime} \cong I / J$ is $\tau$-torsion, and hence, $\tau$-injective. It follows that $I$ is $\tau$-injective, and hence, $I$ is a direct summand of $R$. Therefore, every module is $\tau$-injective by [4, Proposition 8.10].

There are situations when the condition that every cyclic $\tau$-torsion module is $\tau$ injective assures that every module is $\tau$-injective. We present one based on the recent result stating that every Baer module over a commutative domain is projective [6, Theorem 3.4]. Recall that a module $M$ is called $\tau$-projective if $\operatorname{Ext}_{R}^{1}(M, T)=0$ for every $\tau$-torsion module $T$. If $R$ is a commutative domain and $\tau$ is the usual torsion theory in Mod- $R$, then a $\tau$-projective module is called Baer. We need the following easy lemma.

LEMMA 3.4. Every $\tau$-torsion module is $\tau$-injective if and only if every $\tau$-torsion module is $\tau$-projective.

Corollary 3.5. Let $R$ be a commutative domain and $\tau$ the usual torsion theory in Mod- $R$. The following are equivalent:

(i) Every cyclic $\tau$-torsion module is injective.

(ii) Every $\tau$-torsion module is injective.

(iii) Every $\tau$-torsion module is Baer.

(iv) Every module is injective.

(v) $R$ is a field.

Proof. Recall that a module is $\tau$-torsion if and only if every non-zero element $x \in M$ is annihilated by a non-zero ideal. Since $R / I$ is $\tau$-torsion for every non-zero ideal of $R, \tau$-injectivity coincides with usual injectivity.

(i) $\Rightarrow$ (ii) By Corollary 3.1 .

(ii) $\Rightarrow$ (iii) By Lemma 3.4.

(iii) $\Rightarrow$ (iv) By Lemma 3.4, every $\tau$-torsion module is Baer, and so projective by [6, Theorem 3.4]. Then every module is $\tau$-injective [4, Proposition 8.10], and so injective.

(iv) $\Rightarrow$ (v) In this case $R$ is semi-simple, and so $R$ must be a field.

(v) $\Rightarrow$ (i) Clear.

In the following, we establish a characterization of semi-simple modules using certain relative injective modules. Let $\tau$ be a hereditary torsion theory in the category $\sigma[M]$. Recall that a module $N \in \sigma[M]$ is called $(M, \tau)$-injective if $N$ is injective 
with respect to every exact sequence $0 \rightarrow K \rightarrow L$ in $\sigma[M]$ with $L / K \tau$-torsion. We consider the following notion which generalizes that of complemented module with respect to a hereditary torsion theory in Mod- $R$ from [11]. A module $N \in \sigma[M]$ is called $(M, \tau)$-complemented if every submodule of $N$ is $\tau$-dense in a direct summand of $N$.

THEOREM 3.6. The following are equivalent:

(i) $M$ is semi-simple.

(ii) Every module in $\sigma[M]$ is $(M, \tau)$-injective $(M, \tau)$-complemented.

(iii) Every cyclic module in $\sigma[M]$ is $(M, \tau)$-injective $(M, \tau)$-complemented.

(iv) Every cyclic module in $\sigma[M]$ is injective in $\sigma[M]$.

Proof. ( $i) \Rightarrow$ (ii) Suppose that $M$ is semi-simple. Then every module in $\sigma[M]$ is injective in $\sigma[M][\mathbf{1 4}, 20.3]$, and hence, $(M, \tau)$-injective. Also, every module in $\sigma[M]$ is semi-simple in $\sigma[M][\mathbf{1 4}, 20.3]$, and hence, $(M, \tau)$-complemented.

(ii) $\Rightarrow$ (iii) Clear.

(iii) $\Rightarrow($ iv $)$ Let $\mathcal{C}$ be the smallest closed subcategory of $\sigma[M]$ containing the $(M, \tau)$ complemented modules. Then $\mathcal{C}=\sigma[N]$ for some module $N \in \sigma[M]$, and a family of finitely generated generators for $\mathcal{C}$ consists of the modules $R / I$ with $R / I \in \sigma[N]$. Each such $R / I$ is $(M, \tau)$-complemented, and so an object of $\mathcal{C}$. Thus, $\mathcal{C}=\sigma[M]$. By an easy adaptation of [13, Lemma 2] in $\sigma[M]$, it follows that $\tau$ is a generalization of the Goldie torsion theory; hence, $(M, \tau)$-injectivity coincides with injectivity.

(iv) $\Rightarrow$ (i) By Corollary 2.12.

Now we have the following characterization of semi-simple rings.

COROllary 3.7. $R$ is semi-simple if and only if every cyclic module is $\tau$-injective $\tau$-complemented.

The classical Osofsky theorem is obtained by taking $\tau=\tau_{G}$, i.e. the Goldie torsion theory, or $\tau=\chi$, i.e. the torsion theory with all modules torsion. Note that a module is $\tau_{G}$-injective $\tau_{G}$-complemented if and only if it is injective. Also, every module is $\chi$-complemented.

In [1] it has been shown that the class of $\tau$-injective $\tau$-complemented modules is strictly contained in the class of quasi-injective modules. Now recall the following result.

THEOREM 3.8 [7, Theorem 6.83]. The following are equivalent:

(i) $R$ is semi-simple.

(ii) Every module is quasi-injective.

(iii) Every finitely generated module is quasi-injective.

The condition that every cyclic module is quasi-injective is, in general, weaker than that in the previous theorem. For instance, $R=\mathbb{Q}[x] /\left(x^{2}\right)$ is self-injective, and every cyclic module is quasi-injective, but $R$ is not semi-simple [7]. Hence, Corollary 3.7 may be seen as a refinement of Theorem 3.8 for cyclic modules.

ACKNOWLEDGEMENTS. This work was partially supported by the Romanian grants PN-II-ID-PCE-2008-2 project ID_2271, PN-II-ID-PCE-2007-1 project ID_1005 and MEC of Spain. 


\section{REFERENCES}

1. S. Crivei, On $\tau$-complemented modules, Mathematica (Cluj) 45(68) (2003), 127-136.

2. S. E. Dickson, A torsion theory for abelian categories, Trans. Amer. Math. Soc. 121 (1966), 223-235.

3. N. V. Dung, D. V. Huynh, P. F. Smith and R. Wisbauer, Extending modules, Pitman Research Notes in Mathematics Series, vol. 313 (Longman Scientific \& Technical, Harlow, UK, 1994).

4. J. S. Golan, Torsion theories, Pitman Monographs and Surveys in Pure and Applied Mathematics, vol. 29 (Longman Scientific \& Technical, Harlow, UK, 1986).

5. J. L. Gómez Pardo, N. V. Dung and R. Wisbauer, Complete pure injectivity and endomorphism rings, Proc. Amer. Math. Soc. 118 (1993), 1029-1034.

6. L. A. Hügel, S. Bazzoni and D. Herbera, A solution to the Baer splitting problem, Trans. Amer. Math. Soc. 360 (2008), 2409-2421.

7. T. Y. Lam, Lectures on modules and rings (Springer, New York, 1999).

8. B. L. Osofsky, Rings all of whose finitely generated modules are injective, Pacific J. Math. 14 (1964), 645-650.

9. B. L. Osofsky, Noninjective cyclic modules, Proc. Amer. Math. Soc. 19 (1968), $1383-1384$.

10. B. L. Osofsky and P. F. Smith, Cyclic modules whose quotients have all complement submodules direct summands, J. Algebra 139 (1991), 342-354.

11. P. F. Smith, A. M. Viola-Prioli and J. E. Viola-Prioli, Modules complemented with respect to a torsion theory, Comm. Algebra 25 (1997), 1307-1326.

12. B. Stenström, Rings of quotients (Springer-Verlag, Berlin, 1975).

13. A. M. de Viola-Prioli and J. E. Viola-Prioli, The smallest closed subcategory containing the $\mu$-complemented modules, Comm. Algebra 28 (2000), 4971-4980.

14. R. Wisbauer, Foundations of module and ring theory (Gordon and Breach, Reading, UK, 1991). 